\title{
Blackbox Parameter Varying Transfer Functions Model for Highly Nonlinear Electronic Power Converters in DC Microgrids
}

\author{
A. Francés, R. Asensi, and J. Uceda \\ Centro de Electrónica Industrial (CEI) \\ Universidad Politécnica de Madrid \\ Madrid, Spain \\ Email: airan.frances@upm.es
}

\begin{abstract}
The power electronic based de distribution systems are becoming an interesting solution in many applications as electric vehicles (automobiles, planes, ships, etc.), data centers, and microgrids. On the one hand, due to the complexity and the amount of these installations, the use of sophisticated commercialoff-the-shelf converters is very convenient. On the other hand, the use of commercial converters complicates the system-level analysis of these systems, due to the lack of detailed information about the dynamic response of these devices. A solution to this issue is the blackbox modeling approach, which is able to identify behavioral models of the converters from their response to specific tests. However, the approaches available in the literature are not able to account for strong nonlinear dynamic behaviors, such as the ones shown by converters with discontinuous and continuous conductions modes or the ones that integrate different control modes. In this paper, a blackbox Parameter Varying Transfer Functions (PVTF) model is proposed in order to obtain very accurate models of commercial converters in dc microgrid applications.
\end{abstract}

\section{INTRODUCTION}

Modeling Electronic Power Converter (EPC) based systems gives system designers the opportunity to analyze the dynamic performance of the systems in many different scenarios before the actual installation [1]. However, the use of CommercialOff-The-Shelf (COTS) converters makes it difficult to obtain accurate models of the devices, due to confidentiality issues. In these cases, identification techniques can be applied in order to obtain models from the response of the converters to certain perturbations (blackbox modeling). The blackbox modeling approaches can be classified into linear [2], [3], static nonlinear [4], and dynamic nonlinear structures [5], [6], according to the kind of responses that they can reproduce. The strong variability of the dc microgrids often compromises the accuracy of linear models, due to the wide range of operating conditions. In some cases, the nonlinearities of the power converters are mostly reflected in their steady-state response, which can be modeled using Wiener-Hammerstein models. However, in general, the EPCs present an operating point dependent dynamic behavior, so dynamic nonlinear structures such as the polytopic model should be implemented to accurately reproduce their dynamic response.

The polytopic model is able to reproduce the nonlinear dynamic behavior of EPCs when small perturbations are applied in different operating points, when large-signal and slow perturbations are introduced, or when the nonlinearities of the converter are soft. For instance, these structures have been applied to model dc microgrids integrating different converters using system-level control strategies [7]-[9]. However, when the dynamic nonlinearities are strong and the variations of the input variables are sharp, the accuracy of the model is compromised.

This paper proposes a new blackbox modeling approach capable of accounting for EPCs with strong nonlinear dynamic behaviors.

\section{Parameter Varying Transfer Functions Model}

The PVTF model is inspired by the small signal Gparameters model and the polytopic model, which will be briefly described here.

\section{A. G-parameters Model}

The G-parameters model consists of four transfer functions that describe the dynamic response of the output variables when the input variables are perturbed:

$$
\left(\begin{array}{c}
\tilde{v}_{o} \\
\tilde{i}_{i n}
\end{array}\right)=\left(\begin{array}{cc}
G(s) & -Z_{o}(s) \\
Y_{i}(s) & H(s)
\end{array}\right)\left(\begin{array}{c}
\tilde{v}_{i n} \\
\tilde{i}_{o}
\end{array}\right)
$$

where $\tilde{v}_{o}$ and $\tilde{i}_{i n}$ are the small-signal contributions to the output voltage and the input current, respectively, which is produced by the perturbations introduced in the input variables, $\tilde{v}_{i n}$ and $\tilde{i}_{o}$, corresponding to the input voltage and the output current. The transfer functions $G(s), Z_{o}(s), Y_{i}(s)$ and $H(s)$ define the small-signal behavior of the converter around an operating point. These four G-parameters are obtained using the superposition theorem and their physical meaning is represented as follows:

Audio-susceptibility

$$
\text { Output impedance }
$$

$$
G(s)=\left.\frac{\tilde{v}_{o}}{\tilde{v}_{i n}}\right|_{\tilde{i}_{o}=0}
$$$$
Z_{o}(s)=\left.\frac{\tilde{v}_{o}}{\tilde{i}_{o}}\right|_{\tilde{v}_{i n}=0}
$$

Input admittance

$$
\text { Back current gain }
$$

$$
Y_{i}(s)=\left.\frac{\tilde{i}_{i n}}{\tilde{v}_{i n}}\right|_{\tilde{i}_{o}=0} \quad H(s)=\left.\frac{\tilde{i}_{i n}}{\tilde{i}_{o}}\right|_{\tilde{v}_{i n}=0}
$$

The perturbations can be performed in time or frequency domain [3], [6]. In order to identify the transfer functions the 
(a)

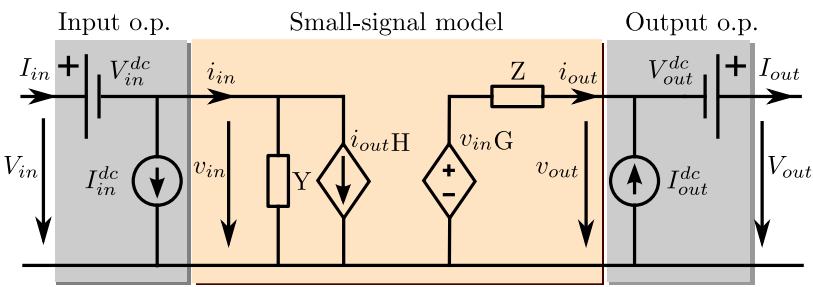

(b)

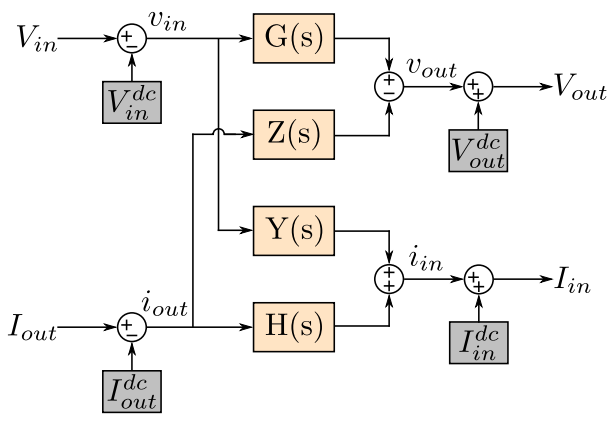

Figure 1: G-parameters model. (a) Equivalent electrical circuit, (b) Block diagram notation.

input perturbation is measured as well as the response of the output variables, according to (2). The data can be imported to a software in order to obtain the transfer functions that reproduce the measured behavior, as the System Identification Toolbox of Matlab [10].

In Fig. 1 the G-parameters model is represented with its equivalent electrical circuit and its block diagram, which could be implemented in any simulation software able to include transfer functions and mathematical operations.

This kind of model can be also derived from the averaged analytical equations of the system after a linearization around an operating point. They have been widely used for dynamic interaction analysis of EPCs-based systems [11]-[13] and stability analysis [14], [15]. However, its accuracy is limited by the small-signal approximation.

\section{B. Polytopic Model}

The polytopic model consists of a weighting combination of small-signal models identified around different operating points:

$$
\begin{aligned}
V_{o} & =\sum_{i=1}^{n} \sum_{j=1}^{m} \omega_{i j}(\alpha, \beta, \ldots) V_{\text {out }}^{i j}\left(V_{\text {in }}, I_{\text {out }}\right) \\
I_{\text {in }} & =\sum_{i=1}^{n} \sum_{j=1}^{m} \omega_{i j}(\alpha, \beta, \ldots) I_{\text {in }}^{i j}\left(V_{\text {in }}, I_{\text {out }}\right)
\end{aligned}
$$

where $V_{\text {out }}$ and $I_{\text {in }}$ are the outputs variables of the polytopic model, corresponding to the output voltage and the input current, $\omega_{i j}$ are the weighting functions, and $V_{o u t}^{i j}$ and $I_{i n}^{i j}$ are the output signals of the local model $i j$. The model structure is depicted in Fig. 2.

Each local model is associated with a particular weighting function, which will be equal to 1 when the input variables are equal to the operating point of its local model and it will be

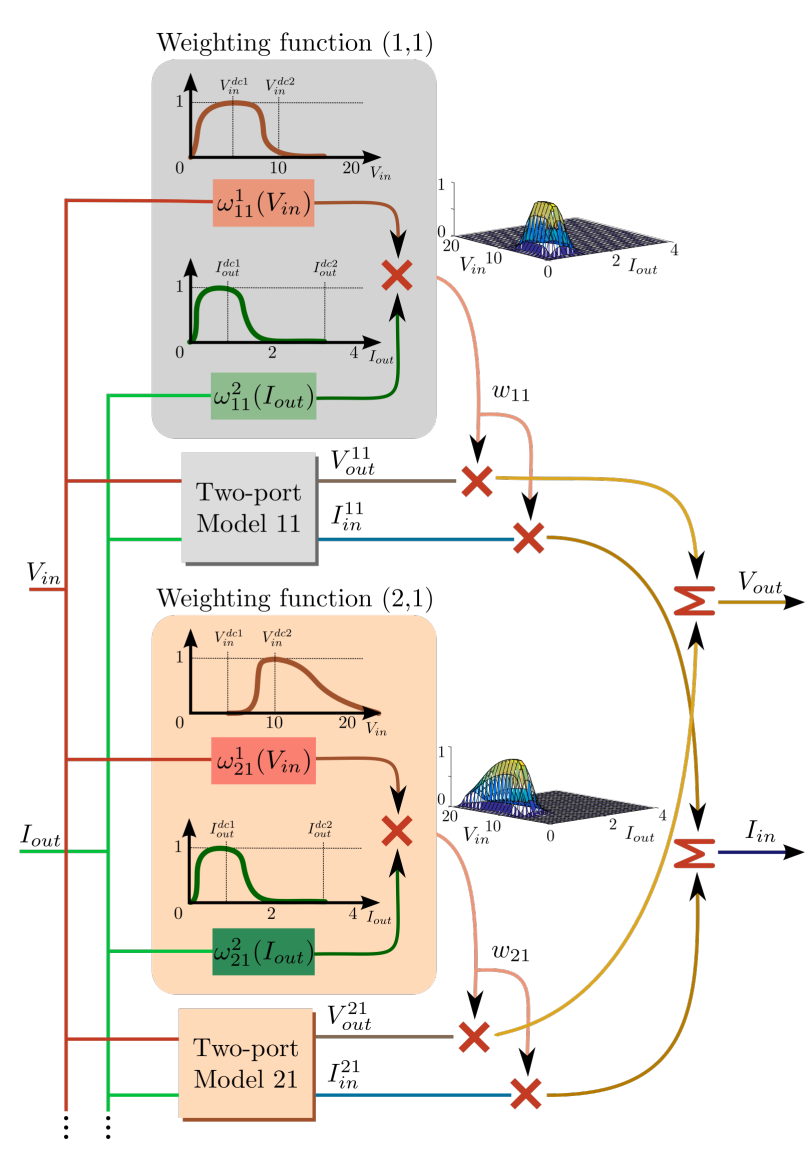

Figure 2: Polytopic model diagram.

equal to 0 when the input variables are far away from the operating point. When the input variables are between two operating points, they will have a value between 0 and 1 such that:

$$
\begin{gathered}
0 \leq \omega_{i j}(\alpha, \beta, \ldots) \leq 1 \\
\sum_{i=1}^{n} \sum_{j=1}^{n} \omega_{i j}(\alpha, \beta, \ldots)=1
\end{gathered}
$$

Typical examples of weighting functions are the triangular, trapezoidal, or sigmoid functions. A common choice of weighting function for this kind of application is the double sigmoid, defined as:

$$
\begin{aligned}
\omega_{i}(\alpha)= & \left(\frac{1}{1+e^{-m_{i}\left(\alpha-c_{i}\right)}}\right)- \\
& \left(\frac{1}{1+e^{-m_{i+1}\left(\alpha-c_{i+1}\right)}}\right)
\end{aligned}
$$

where $m_{i}$ and $c_{i}$ are the slope and the center of the rising edge of the sigmoid and $m_{i+1}$ and $c_{i+1}$ are the slope and the center of the falling edge. Notice that the adjacent weighting functions must have the same center and slope in order to ensure the second constrain shown in (5). Consequently, a multidimensional weighting function can be built as the product of unidimensional ones: 


$$
\omega_{i j}(\alpha, \beta, \ldots)=\omega_{i}(\alpha) \omega_{j}(\beta) \ldots
$$

This large-signal blackbox modeling approach has been successfully applied to EPCs with operating point dependent dynamic behaviors [5], [16] and, to some extent, for large-signal stability analysis [17]. However, in case the input variables change with sharp steps, the accuracy of this approximation can be compromised during the transient response.

\section{Dynamic Weighting Functions}

The traditional weighting functions described before, (6), are static functions depending on the input variables. Therefore, they establish a relationship between the dynamic behavior of each local model and the dynamic behavior of the overall model according to the current value of the input variables. Consequently, a sharp step in the input variables will lead to a sharp transition in the dynamic response of the model from the one defined by the initial operating point to the one defined by the final operating point. Nevertheless, the dynamic of a system is related with its state variables, which have dynamic restrictions (for instance, capacitor voltages and inductor currents). In [18] the use of dynamic weighting functions was proposed in order to include this dynamic limitation in the transition among local models.

The approach is to include a transfer function between the input variables and the input of the weighting functions, such that the effective input of the weighting functions is filtered version of the input variables. Therefore, the dynamic weighting functions can be expressed as:

$$
\omega_{i j}^{d}(\alpha, \beta, \ldots, s)=\omega_{i}\left(\alpha \tau_{\alpha}(s)\right) \cdot \omega_{j}\left(\beta \tau_{\beta}(s)\right) \ldots
$$

where $\omega_{i j}^{d}(\alpha, \beta, \ldots, s)$ are the dynamic weighting functions of the local models $i j, \omega_{i}$ and $\omega_{j}$ are the static weighting functions defined in (7), and $\tau_{\alpha}(s)$ and $\tau_{\beta}(s)$ are the transfer functions that limit the dynamic transition among local models. $\tau_{\alpha}(s)$ and $\tau_{\beta}(s)$ can be designed using the poles of the system, which have the information about its dynamic, assuring that their steady-state gain is equal to 1 , so they do not affect the stationary response. Hence, these transfer functions can be expressed as follows:

$$
\tau(s)=\frac{a_{0}}{s^{n}+a_{n-1} s^{n-1}+\ldots+a_{1} s+a_{0}}
$$

where the denominator is the characteristic polynomial of the system, thus named characteristic filter. This filter could also be a weighted combination of local filter, as the characteristic polynomial can depend on the operating point. This methodology has been applied to EPCs with nonlinear dynamic behavior when the inputs change sharply showing a high accuracy [9], [18]. However, in case the dynamic behavior of the different small-signal models is substantially different, the accuracy of this approach can also be compromised. The main reason for the loss of accuracy of the polytopic model with dynamic weighting functions in this scenario is related with the initial conditions of the small-signal models during the transient response. As the local models are independent of each other, in case their dynamics are very different, the transition among their output response can lead to discontinuities.
In order to solve this problem, instead of using independent small-signal models, a single G-parameters structure is proposed, where the coefficients of the transfer functions are variable. The construction of the PVTF follows a similar methodology to the polytopic model. Different local models are identified around different operating points and dynamic weighting functions are used to modify the parameters of the transfer functions that integrate the G-parameters model according to the operating point.

$$
\left(\begin{array}{c}
\tilde{v}_{o} \\
\tilde{i}_{i n}
\end{array}\right)=\left(\begin{array}{cc}
G(\alpha, \beta, s) & -Z_{o}(\alpha, \beta, s) \\
Y_{i}(\alpha, \beta, s) & H(\alpha, \beta, s)
\end{array}\right)\left(\begin{array}{c}
\tilde{v}_{i n} \\
\tilde{i}_{o}
\end{array}\right)
$$

where $G(\alpha, \beta, s), Z_{o}(\alpha, \beta, s), Y_{i}(\alpha, \beta, s)$, and $H(\alpha, \beta, s)$ are transfer function with variable parameters that depend on the operating point. A general transfer function that depends on one variable, $\alpha$, could be represented as:

$$
G(\alpha, s)=\frac{b_{n}(\alpha, s) s^{n}+\ldots+b_{1}(\alpha, s) s+b_{0}(\alpha, s)}{s^{n}+a_{n-1}(\alpha, s) s^{n-1}+\ldots+a_{1}(\alpha, s) s+a_{0}(\alpha, s)}
$$

where the parameters $b_{j}(\alpha, s)$ and $a_{j}(\alpha, s)$ are defined as follows:

$$
\begin{aligned}
& b_{j}(\alpha, s)=\sum_{i=1}^{n} \omega_{i}^{d}(\alpha, s) b_{j}^{i} \\
& a_{j}(\alpha, s)=\sum_{i=1}^{n} \omega_{i}^{d}(\alpha, s) a_{j}^{i}
\end{aligned}
$$

where $\omega_{i}^{d}(\alpha, s)$ are the dynamic weighting functions described in (8), $i$ represent the different local models, and $j$ the different parameters. Equivalently, the transfer function can be expressed in the gain, zero, pole format and vary these parameters in order to work with meaningful information.

The methodology to implement the proposed model will be described with an example. Consider the following transfer function with one zero and two complex poles:

$$
\frac{y(z)}{u(z)}=G(z)=\frac{K\left(z-Z_{1}\right)}{(z-R+j I)((z-R-j I))}
$$

where $K$ is the gain, $Z_{1}$ is the zero, and $R$ and $I$ are the real and imaginary parts of the complex poles. In order to implement this method in a simulator, it is more convenient to use discrete domain, because changing the parameter of continuous transfer functions can easily lead to instabilities. Besides, in order to be able to vary the parameters freely, the transfer function can be rearranged as follows:

$$
\begin{aligned}
y(z)= & K u(z) z^{-1}-Z_{1} K u(z) z^{-2}+2 R y(z) z^{-1}- \\
& -\left(R^{2}+I^{2}\right) y(z) z^{-2}
\end{aligned}
$$

This expression can be easily implemented in any simulator that includes delays and mathematical operations, as represented in Fig. 3. The overall model of the parameter varying transfer function includes the blocks that vary the parameters $\left(K, Z_{1}, R\right.$ and $\left.I\right)$ depicted in Fig. 4. The gray box represents the static part of the weighting functions, it 


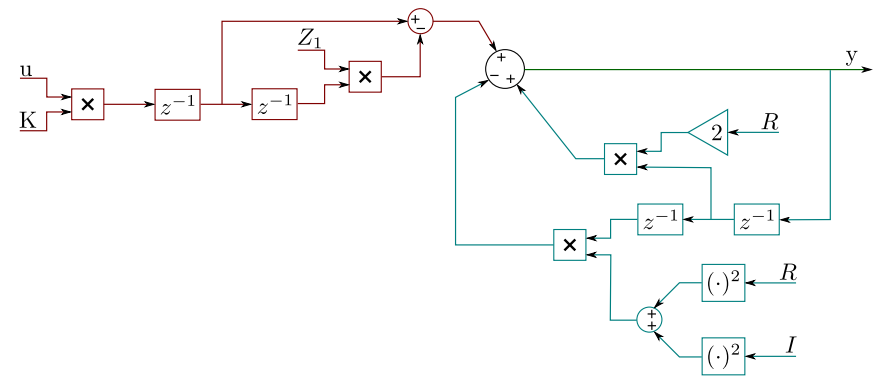

Figure 3: Block diagram of a parameter varying transfer function, $G(z)$, with one zero and a pair of complex-conjugate poles.

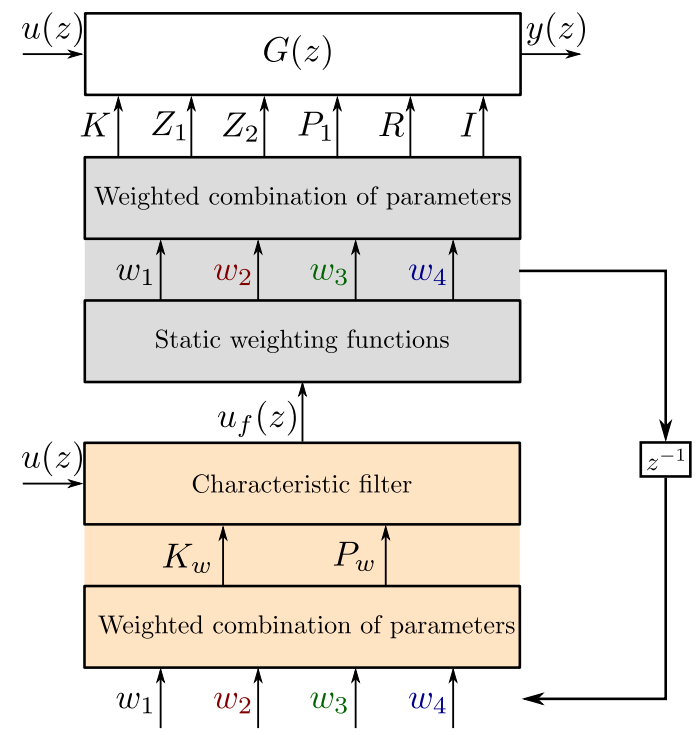

Figure 4: Block diagram of the parameter varying transfer functions model.

receives the filtered input signal and produces the weighted combination of the parameters for the transfer function, as depicted in detail in Fig. 5. The brown box represents the characteristic filter that introduces dynamic in the weighting function. This characteristic filter is also considered variable and depending on the poles of the system in different operating points, as depicted in Fig. 6.

The advantage of the PVTF model in comparison with the polytopic model is that when the dynamic of the model varies it takes into account the past values of the input and output variables, instead of weighting the contribution of independent local models. Consequently, it will provide a better approximation of the transient response of highly nonlinear EPCs to sharp changes in the input variables.

\section{SYSTEM DESCRIPTION}

The proposed model will be validated with a buck converter working in continuous and discontinuous conduction modes. In particular, the transition between these conduction modes will be studied. Notice that in discontinuous conduction mode the behavior of the converter is highly nonlinear and substantially different from the behavior in continuous conduction mode. In this scenario, the most relevant input variable is the output
Static weighting fucntions

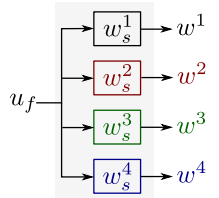

Weighted combination of parameters

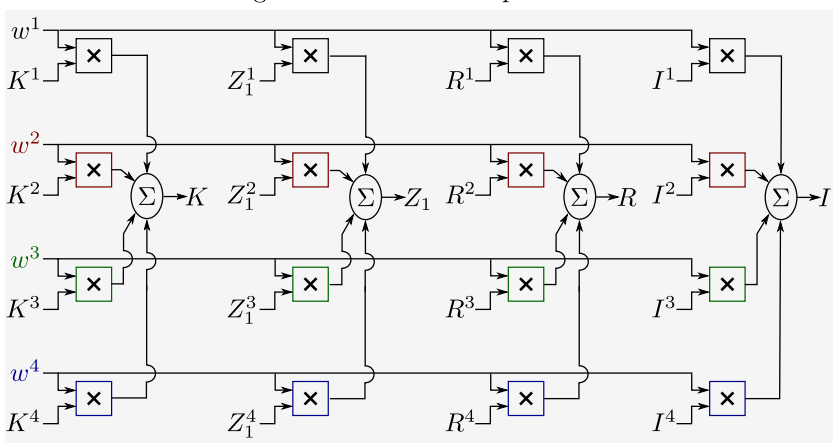

Figure 5: Block diagram of the weighted combination of local parameters.
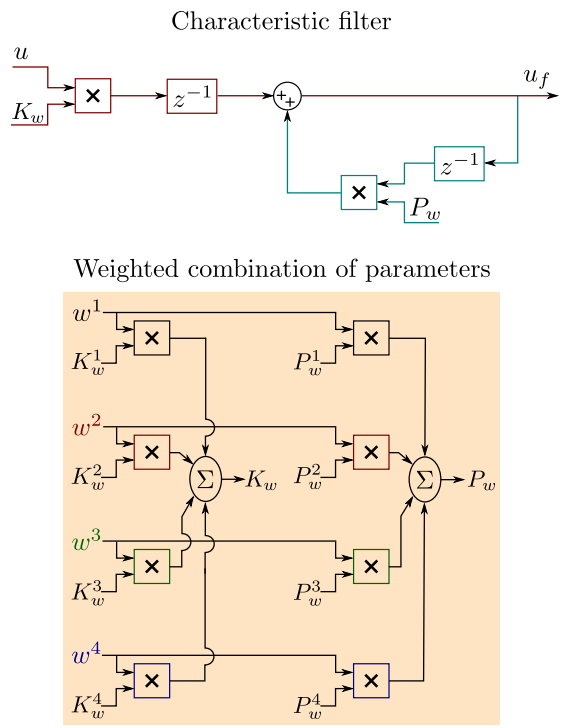

Figure 6: Block diagram of the characteristic filter.

current, which can vary in a wide range depending on the load. The discontinuous conduction mode will appear in light load conditions, when the inductor is discharged and the current cannot flow towards the source during part of the switching cycle. At heavy load conditions, the average inductor current value will be high enough so the current ripple is above zero.

The experimental setup (Fig. 7) consists of a dc power supply (24 V) and an electronic load working as a constant current load. The buck converter control has an inner current loop and an output voltage loop $(12 \mathrm{~V})$. The control and monitoring of the system are performed by a Dspace Scalexio unit, which integrates an FPGA able to generate the PWM signal $(10 \mathrm{kHz})$ and the inner control loop. The outer control loop is implemented in the processor part, which can be modified in real-time using the control desk. An oscilloscope will be used to record the results of the tests. 


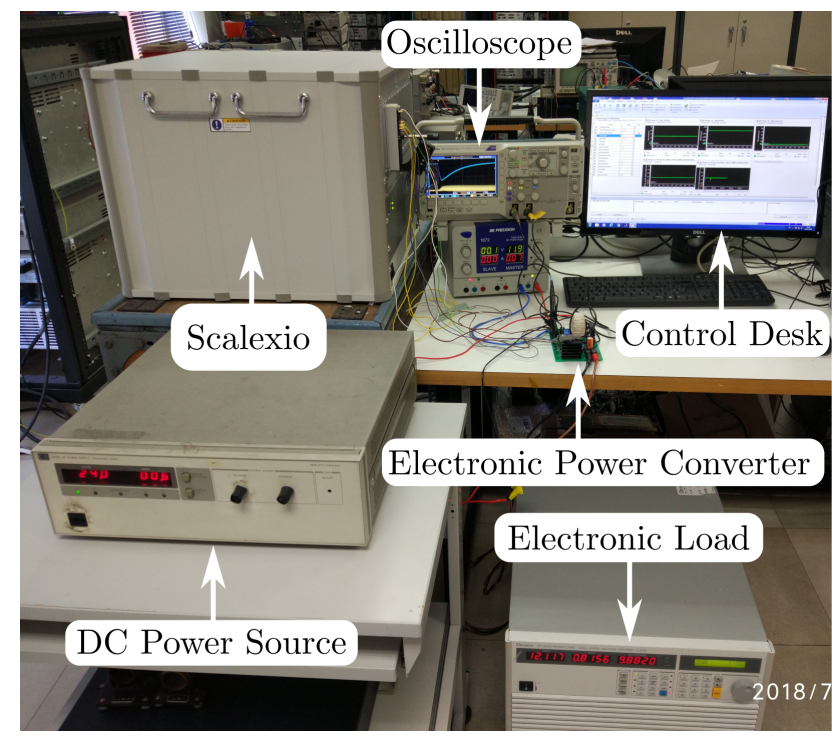

Figure 7: Experimental Setup.

\section{Model IdentificATION}

In this section, the identification of the output impedance will be detailed, which is the most relevant G-parameter in this case. The transfer function related to the output impedance describes the behavior of the output voltage when the output current is perturbed.

In order to obtain the small-signal models, the electronic load is used to perform small steps in the output current, which is able to provide a slew rate high enough to obtain information in the range of frequencies of interest. Seven smallsignal models were identified for values of the output current from $0.1 \mathrm{~A}$ to $0.7 \mathrm{~A}$, where the steps had an amplitude of $0.1 \mathrm{~A}$. The step is given to the output current and the response of the output voltage is measured, conditioned, and imported to the System Identification Tool of Matlab, which provides the transfer functions that approximate the small-signal behavior of the output impedance of the converter. Afterward, using model order reduction techniques, second-order transfer functions were obtained from the tests performed. The identified transfer functions are presented in Table I, and the placement of the poles according to the operating point of the output current is depicted in Fig. 8. It can be seen how for values of the output current below 0.3 A the system has complex poles, whereas for higher values the poles become real. All the models have a zero in the origin because the output voltage is regulated. Above 0.7 A the converter works in continuous conduction mode.

The general transfer function able to include all the smallsignal models identified is:

$$
Z_{o}(z)=\frac{K\left(z-z_{1}\right)}{z^{2}+\left(p_{1}+p_{2}\right) z+\left(p_{1} p_{2}+I^{2}\right)}
$$

where $Z_{o}(z)$ is the discrete time transfer function of the output impedance, $z_{1}$ is the zero which will always be equal to $1, K$ is the gain, $p_{1}$ and $p_{2}$ are the real part of the poles, and $I$ is the imaginary part of the poles. Notice that in case the poles are real, $I$ is equal to zero, whereas if the poles are complex,
Table I: Identified transfer functions for different values of the output current.

\begin{tabular}{|c||c|}
\hline Output Current & Transfer function \\
\hline \hline $0.1 A$ & $\frac{-2278.4 s}{(s+30.21+34.63 i)(s+30.21-34.63 i)}$ \\
\hline $0.2 A$ & $\frac{-2226.2 s}{(s+30.21+34.63 i)(s+30.21-34.63 i)}$ \\
\hline $0.3 A$ & $\frac{-2188.4 s}{(s+67.44)(s+41.89)}$ \\
\hline $0.4 A$ & $\frac{-2165.9 s}{(s+115.9)(s+28.7)}$ \\
\hline $0.5 A$ & $\frac{-2086.3 s}{(s+127.5)(s+25.44)}$ \\
\hline $0.6 A$ & $\frac{-2175.5 s}{(s+184.9)(s+19.77)}$ \\
\hline $0.7 A$ & $\frac{-2074.7 s}{(s+194.1)(s+19.89)}$ \\
\hline
\end{tabular}

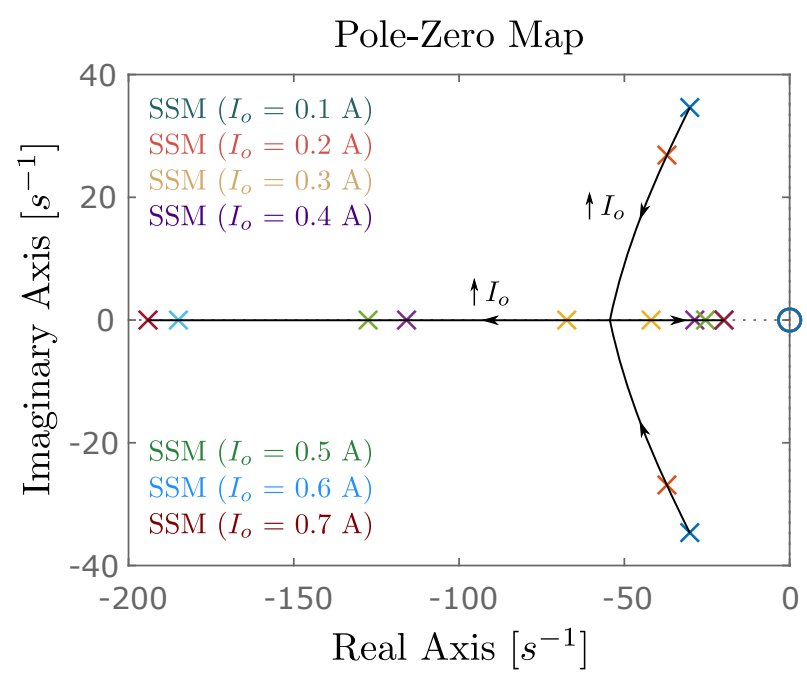

Figure 8: Poles placement according to the output current.

$p_{1}=p_{2}=R$. This transfer function can be represented in the following form, which is very suitable to create a PVTF model:

$V_{o}=I_{o} K z^{-1}-z_{1} I_{o} K z^{-2}+\left(p_{1}+p_{2}\right) V_{o} z^{-1}-\left(p_{1} p_{2}+I^{2}\right) V_{o} z^{-2}$

Notice that in case $p_{1}=p_{2}=R$ this equation is equal to the one described in (14). This expression can be implemented in any software that includes signals, parameters, delay blocks, and multipliers, see Fig. 9. The parameters $\left(K, z_{1}, p_{1}, p_{2}, I\right)$ will be the ones identified in the small-signal models and they will be varied according to the operating point of the output current, as described in Section II.

\section{EXPERIMENTAL VALIDATION}

The performance of the PVTF model is compared with the response of the system to a transition from DCM to CCM. In Fig. 4 the measured signal from the buck converter is 


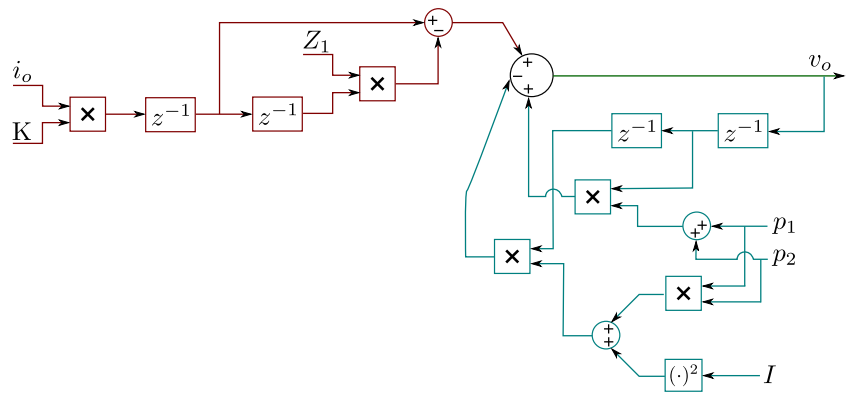

Figure 9: Scheme of the PVTF model implemented.
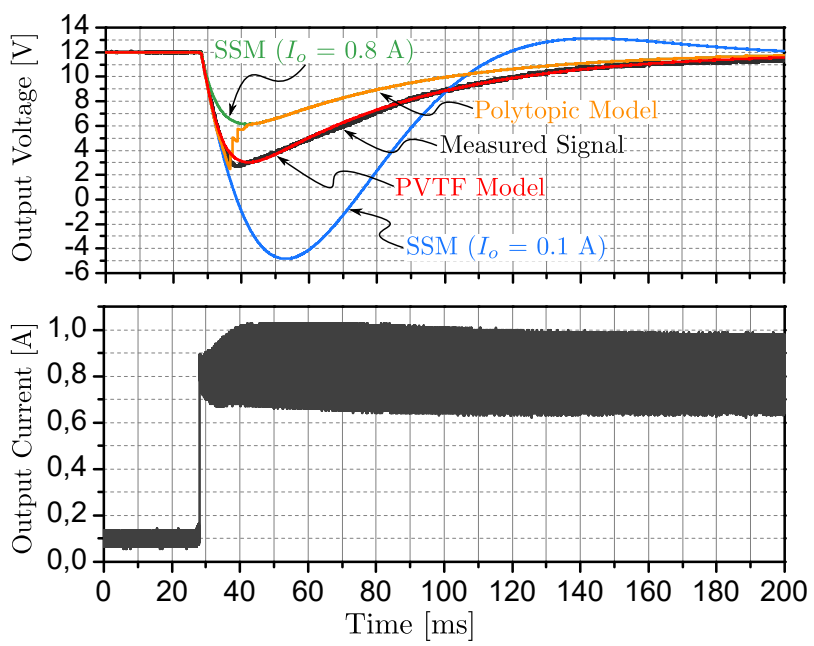

Figure 10: Comparison between the actual converter response and the small-signal models (SSM) and the Parameter Varying Transfer Functions (PVTF) model for a transition between DCM and CCM.

compared with the PVTF, two of the small-signal models identified, corresponding to the initial and the final operating point, and the polytopic model. At time $t=28 \mathrm{~ms}$ a load step is performed changing the output current from $i_{o}=0.1 \mathrm{~A}$ to $i_{o}=0.8 \mathrm{~A}$. It can be seen that the accuracy of the proposed model is substantially higher than the one obtained with the small-signal models. The polytopic model makes a transition among the different small-signal models, but due to the strong difference in the dynamic behavior of the converter, the resulting response is discontinuous and its performance is poor for this kind of transition.

\section{CONCLUSIONS}

This paper proposes a novel blackbox modeling technique able to reproduce the response of dc electronic power converters with strong nonlinearities. The model is based on existing approaches, G-parameters and polytopic models, which have been described. The proposed method is able to modify the dynamic behavior of the system taking into account the past values of the input and output variables. This capability allows the model to account for strong nonlinearities. A buck converter in DCM and CCM has been used to validate the model. The identification process and a practical model construction have been detailed. The performance of the proposed modeling technique is compared with the existing solutions.

\section{ACKNOWLEDGMENT}

This work was supported by the Spanish Ministry of Economy and Competitiveness under the project IDENMRED with reference DPI2016-78644-P.

\section{REFERENCES}

[1] A. Frances, R. Asensi, O. Garcia, R. Prieto, and J. Uceda, "Modeling electronic power converters in smart dc microgrids-an overview," IEEE Transactions on Smart Grid, vol. 9, no. 6, pp. 6274-6287, Nov 2018.

[2] L. Arnedo, D. Boroyevich, R. Burgos, and F. Wang, "Un-terminated frequency response measurements and model order reduction for blackbox terminal characterization models," in Proc. Appl. Power Electron. Conf. Expo., Feb 2008, pp. 1054-1060.

[3] I. Cvetkovic, D. Boroyevich, P. Mattavelli, F. C. Lee, and D. Dong, "Unterminated small-signal behavioral model of dc-dc converters," IEEE Trans. Power Electron., vol. 28, no. 4, pp. 1870-1879, April 2013.

[4] J. Oliver, R. Prieto, J. Cobos, O. Garcia, and P. Alou, "Hybrid wienerhammerstein structure for grey-box modeling of dc-dc converters," in Proc. Appl. Power Electron. Conf. Expo., Feb 2009, pp. 280-285.

[5] L. Arnedo, D. Boroyevich, R. Burgos, and F. Wang, "Polytopic blackbox modeling of dc-dc converters," in Proc. IEEE Annu. Power Electron. Spec. Conf., June 2008, pp. 1015-1021.

[6] V. Valdivia, A. Barrado, A. Roldan, C. Fernandez, and P. Zumel, "Blackbox modeling of $\mathrm{dc}-\mathrm{dc}$ converters based on transient response analysis and parametric identification methods," in Proc. Appl. Power Electron. Conf. Expo., Feb 2010, pp. 1131-1138.

[7] A. Francés, R. Asensi, O. García, R. Prieto, and J. Uceda, "A Black-box Modeling Approach for DC Nanogrids," in Proc. Appl. Power Electron. Conf. Expo., 2016, pp. 1624-1631.

[8] - "The performance of polytopic models in smart dc microgrids," in Proc. IEEE Energy Conversion Congr. Exposition, Sept 2016, pp. 1-8.

[9] A. Frances-Roger, A. Anvari-Moghaddam, E. Rodriguez-Diaz, J. C. Vasquez, J. M. Guerrero, and J. Uceda, "Dynamic assessment of cots converters-based dc integrated power systems in electric ships," IEEE Transactions on Industrial Informatics, pp. 1-1, 2018.

[10] MathWorks, "System Identification Toolbox User's Guide," p. 1164, 2016. [Online]. Available: https://es.mathworks.com/help/pdf_doc/ident/ident.pdf

[11] M. Hankaniemi, M. Karppanen, T. Suntio, A. Altowati, and K. Zenger, "Source-reflected load interactions in a regulated converter," in Proc. IEEE Annu. Conf. Ind. Electron. Soc., Nov 2006, pp. 2893-2898.

[12] T. Suntio, M. Hankaniemi, and M. Karppanen, "Analysing the dynamics of regulated converters," IET IEE Proc. Electric Power Applicat., vol. 153, no. 6, pp. 905-910, November 2006.

[13] J. Leppäaho, J. Huusari, L. Nousiainen, and T. Suntio, "Dynamics of current-fed converters and stability-assessment of solar-generator interfacing," in Proc. IEEE Int. Power Electron. Conf., June 2010, pp. 703 709 .

[14] M. Veerachary and A. R. Saxena, "G-parameter based stability analysis of dc-dc power electronic system," in Proc. IEEE Joint Int. Conf. Power Syst. Technology Power India Conf., Oct 2008, pp. 1-4.

[15] S. Vesti, T. Suntio, J. A. Oliver, R. Prieto, and J. A. Cobos, "Impedancebased stability and transient-performance assessment applying maximum peak criteria," IEEE Trans. Power Electron., vol. 28, no. 5, pp. 2099 2104, May 2013.

[16] L. Arnedo, R. Burgos, D. Boroyevich, and F. Wang, "System-level blackbox dc-to-dc converter models," in 2009 Twenty-Fourth Annual IEEE Applied Power Electronics Conference and Exposition, Feb 2009, pp. 1476-1481.

[17] A. Francés, R. Asensi, O. García, and J. Uceda, "A blackbox large signal lyapunov-based stability analysis method for power converterbased systems," in Proc. IEEE Workshop Control Modeling Power Electron., June 2016, pp. 1-6.

[18] A. Francés, R. Asensi, O. García, R. Prieto, and J. Uceda, "How to model a dc microgrid: Towards an automated solution," in Proc. IEEE Second Int. Conf. DC Microgrids, June 2017, pp. 609-616. 